\title{
Sicherung der Lebensgrundlage Grundwasser - eine globale Gemeinschaftsaufgabe
}

\author{
Ralph Watzel ${ }^{1}$
}

Eingegangen: 28. April 2020 / Überarbeitet: 28. April 2020 / Online publiziert: 17. Juni 2020

(c) Der/die Autor(en) 2020

Grundwasser ist sowohl elementare Lebensgrundlage als auch Wirtschaftsgut. Globale Megatrends wie die wachsende Weltbevölkerung, die wirtschaftliche Entwicklung und der Wohlstandszuwachs insbesondere in Schwellenländern in Verbindung mit der Globalisierung der Wertschöpfungsketten erfordern stärkere Anstrengungen zur Sicherstellung der Versorgung mit Wasser und Nahrungsmitteln. Hinzu kommen die Auswirkungen, die der gestiegene Bedarf nach Rohstoffen sowie der Klimawandel auf die Grundwasservorkommen und deren nachhaltige Nutzung haben. Diese Entwicklungen, die wir summarisch als „Global Change“ bezeichnen, führen zu einem erheblichen Nutzungsdruck auf natürliche Ressourcen und einer Verschärfung der damit verbundenen sozialen Fragen. Durch unsere globalisierte Lebensweise und die damit einhergehenden multifaktoriellen Abhängigkeiten werden zukünftig auch scheinbar wasserreiche Regionen von den Auswirkungen der Globalisierung betroffen sein. Mancherorts ist dies in Deutschland bereits heute deutlich spürbar. Die Begrenztheit nutzbarer Grundwasservorkommen ist in ariden und semiariden $\mathrm{Ge}-$ bieten schon heute eine Herausforderung für Politik, Wirtschaft und Gesellschaft. Mittlerweile wird sie auch hierzulande zunehmend Nicht-Fachleuten bewusst.

Grundwasser ist weltweit ein Schlüsselelement der Wasserversorgung, insbesondere der Trinkwasserversorgung. Die Bundesanstalt für Geowissenschaften und Rohstoffe (BGR) ist seit Jahrzehnten in einschlägigen internationalen Projekten engagiert. Im Licht der Herausforderungen des „Global Change“ und basierend auf der jahrzehntelangen Erfahrung bei der Erkundung, Bewertung und Nutzung von Grundwasservorkommen hat die BGR ihre Handlungsschwerpunkte strategisch ausgerichtet. In den kommenden Jahren werden wir uns in Bezug auf das Grundwasser insbesondere mit den Themen Versorgungsicherheit sowie

Ralph Watzel

ralph.watzel@bgr.de

1 Bundesanstalt für Geowissenschaften und Rohstoffe, Stilleweg 2, 30655 Hannover, Deutschland
Dynamik und Stoffumsatz von Grundwasserfließsystemen befassen - auch in Verbindung mit den dafür erforderlichen Flächen- und Rauminformationen. In der internationalen Zusammenarbeit stehen beim Thema Versorgungssicherheit die Entwicklung von Erkundungs- und Nutzungsstrategien für aride Gebiete und Küstenzonen sowie die Methodenentwicklung von Prognosewerkzeugen im Vordergrund. Die BGR sieht in diesen Handlungsfeldern einen drängenden Bedarf hinsichtlich einer präziseren Erfassung von verfügbaren Grundwassermengen und deren Beschaffenheit sowie der Bereitstellung belastbarer Prognosen zur Bewältigung der anstehenden Herausforderungen. Zudem befassen wir uns mit den geowissenschaftlichen Aspekten von Bergbaufolgen. Die BGR gründet in diesem Jahr ein „Forschungs- und Entwicklungszentrum Bergbaufolgen“, das zur nachhaltigen Gestaltung von Bergbaufolgelandschaften Grundwasser- und Bodenbelange erforschen und Technologien weiterentwickeln wird - auch in Form von Forschungskooperationen mit einer internationalen Perspektive.

Die qualifizierte Unterstützung von Entscheidungsprozessen in Politik, Wirtschaft und Gesellschaft erfordert belastbare wissenschaftliche Informationen und Beratung auf höchstmöglichem Niveau. Ein wesentliches Element davon sind auch gut ausgebildete Menschen mit Fachwissen und Kreativität, die hydrogeologische Techniken qualifiziert anwenden, diese weiterentwickeln und Innovationen anstoBen. Wichtige Bausteine unseres strategischen Handelns sind zudem die Zusammenarbeit mit den Staatlichen Geologischen Diensten in Deutschland, Europa und in Übersee, die Kooperation mit Hochschulen und außeruniversitären Forschungseinrichtungen, wissenschaftlichen Gesellschaften und Fachorganisationen sowie die Beteiligung in Gremien und Normungsausschüssen auf nationaler und internationaler Ebene. Die BGR wird ihrer Kompetenz und Erfahrung in Zukunft noch stärker in diese Netzwerke einbringen, um den globalen Herausforderungen zielgerichtet zu begegnen. Die weltweit dauerhafte Sicherung der Lebensgrundlage Grundwasser kann nur partnerschaftlich gelingen. 
Funding Open Access funding provided by Projekt DEAL.

Open Access Dieser Artikel wird unter der Creative Commons Namensnennung 4.0 International Lizenz veröffentlicht, welche die Nutzung, Vervielfältigung, Bearbeitung, Verbreitung und Wiedergabe in jeglichem Medium und Format erlaubt, sofern Sie den/die ursprünglichen Autor(en) und die Quelle ordnungsgemäß nennen, einen Link zur Creative Commons Lizenz beifügen und angeben, ob Änderungen vorgenommen wurden.
Die in diesem Artikel enthaltenen Bilder und sonstiges Drittmaterial unterliegen ebenfalls der genannten Creative Commons Lizenz, sofern sich aus der Abbildungslegende nichts anderes ergibt. Sofern das betreffende Material nicht unter der genannten Creative Commons Lizenz steht und die betreffende Handlung nicht nach gesetzlichen Vorschriften erlaubt ist, ist für die oben aufgeführten Weiterverwendungen des Materials die Einwilligung des jeweiligen Rechteinhabers einzuholen.

Weitere Details zur Lizenz entnehmen Sie bitte der Lizenzinformation auf http://creativecommons.org/licenses/by/4.0/deed.de. 